\title{
Electronic transport of molecular systems
}

\author{
J. Chen, M.A. Reed* \\ Departments of Electrical Engineering, Applied Physics, and Physics, Yale University, New Haven, CT 06520, USA
}

Received 25 April 2002

\begin{abstract}
We report stable and reproducible switching and memory effects in self-assembled monolayers (SAMs). We demonstrate realization of negative differential resistance (NDR) and charge storage in electronic devices that utilize single redox-center-contained SAM as the active component; and compare the effects of various redox centers to switching and storage behavior. The devices exhibit electronically programmable and erasable memory bits with bit retention times greater than $15 \mathrm{~min}$ at room temperature. (c) 2002 Elsevier Science B.V. All rights reserved.
\end{abstract}

\section{Introduction}

The ability to utilize single molecules that function as self-contained electronic devices has motivated researchers around the world for years, concurrent with the continuous drive to minimize electronic circuit elements in semiconductor industry. The microelectronics industry is presently close to the limit of this minimization trend dictated by both laws of physics and the cost of production. It is possible that electronically functional molecular components can not only address the ultimate limits of possible miniaturization, but also provide promising new methodologies for novel architectures, as well as non-linear devices, and memories.

Molecular electronics is conceptually different from conventional solid state semiconductor electronics. It allows chemical engineering of organic molecules with their physical and electronic prop-

\footnotetext{
${ }^{*}$ Corresponding author. Fax: +1-203-432-6420.

E-mail address: mark.reed@yale.edu (M.A. Reed).
}

erties tailored by synthetic methods, bringing a new dimension in design flexibility that does not exist in typical inorganic electronic materials. It is well known that semiconductor devices are fabricated from the "top-down" approach that employs a variety of sophisticated lithographic and etch techniques to pattern a substrate and this approach has become increasingly challenging as feature size decreases. In particular, at nanometer scale, the electronic properties of semiconductor structures fabricated via conventional lithographic processes are increasingly difficult to control. In contrast, molecules are synthesized from the "bottom-up" approach that builds small structures from the atomic, molecular, or single device level. It in principle allows a very precise positioning of collections of atoms or molecules with specific functionalities. For example, one can selectively add an oxygen atom to a molecule with a precision far greater than an oxidation step in microfabrication using the state of the art lithography and etching. Chemical synthesis makes it possible to make large 
quantities of nanometer-size molecules with the same uniformity but at significantly less cost, compared to other batch-fabrication processes such as microlithography. One can envision that in assembling molecular circuits, instead of building individual components on a chip one will synthesize molecules with structures possessing desired electronic configurations and attach/interconnect them into an electronic circuit using surface attachment techniques like self-assembly.

Electronic switching and memory effects are known to exist in a wide variety of inorganic and organic materials. In 1979, Potember et al. [1] reported bistable, reproducible, and nanosecond electronic switching and memory phenomena in an organometallic charge-transfer (CT) complex salt CuTCNQ formed by TCNQ (7, 7, 8, 8-tetracyano$p$-quinodimethane), which acts as an electron acceptor with metallic copper as the electron-rich donor. In these materials the switching and memory phenomena are given rise to by the field assisted structural changes such as phase transitions, crystallization, and metal filament formation assisted by highly localized Joule heating. However, the electronic behavior of these materials is mostly not stable or reproducible, and usually requires high switching voltage [2].

In this paper we discuss stable and reproducible switching and memory effects in SAMs. We first present results on conformation-induced transition behavior; then demonstrate the realization of stable and reproducible large negative differential resistance (NDR) and charge storage in electronic devices that utilize single redox-center-contained SAM as the active component. The devices exhibit electronically programmable and erasable memory bits with bit retention times greater than 15 min at room temperature.

\section{Conductor-insulator transition caused by molec- ular conformation}

Molecular conformation ${ }^{1}$ is one of the most important aspects of chemistry. When molecular

\footnotetext{
${ }^{1}$ Structures that differ only by rotation about one or more bonds are defined as conformations of a compound.
}

systems have sufficient degrees of freedom to adopt a variety of shapes or forms, some of these forms can be associated with stretching or bending of bonds. For example, studies on mechanical distortions of bent nanotubes and crossing nanotubes show that they can exhibit substantial tubular deformations as well as bending and buckling, a factor in turn influencing their electronic transport characteristics [3].

On the other hand, molecular conformation is often associated with rotations of substituents around specific bonds. Zhou [4] observed that there exists a transition temperature below which the benzene rings of a tolane-like molecule assumes a perpendicular configuration, but a coplanar one above the transition temperature. These devices are nanoscale $\mathrm{Au} / \mathrm{Ti}$ /ethyl-substituted 4,4'-di(phenylene-ethynylene)-benzothiolate/ $\mathrm{Au}$ junctions, fabricated using an inverted nanopore structure [4]. The experiments show that current first decreases gradually as temperature varies from room temperature to $30 \mathrm{~K}$ due to thermal activation, then a sharp decrease of two orders of magnitude in conductance resembling a transitional behavior is observed around 25 K (Fig. 1). Calculations by Seminario et al. show that the relative angle between two benzene rings in each tolane molecule determines its conductivity, being maximum at $0^{\circ}$ and minimum at $90^{\circ}$ [5]. At $10 \mathrm{~K}$, phenyl rings in the tolane molecules show very little tendency to rotate and perpendicular tolanes are more stable than parallel tolane molecules-giving rise to smaller conductivity; at $30 \mathrm{~K}$, the tolane rings are able to freely rotate with respect to each other, allowing the tolane molecules to be planar at some instant in time, consequently reaching higher conductivity.

\section{NDR in molecular junctions}

The discovery of NDR in semiconductor diodes has opened a new chapter in semiconductor device physics and device development [5-11]. Through the use of NDR devices, circuits with complicated functions can be implemented with significantly fewer components [12-17]. There are various 


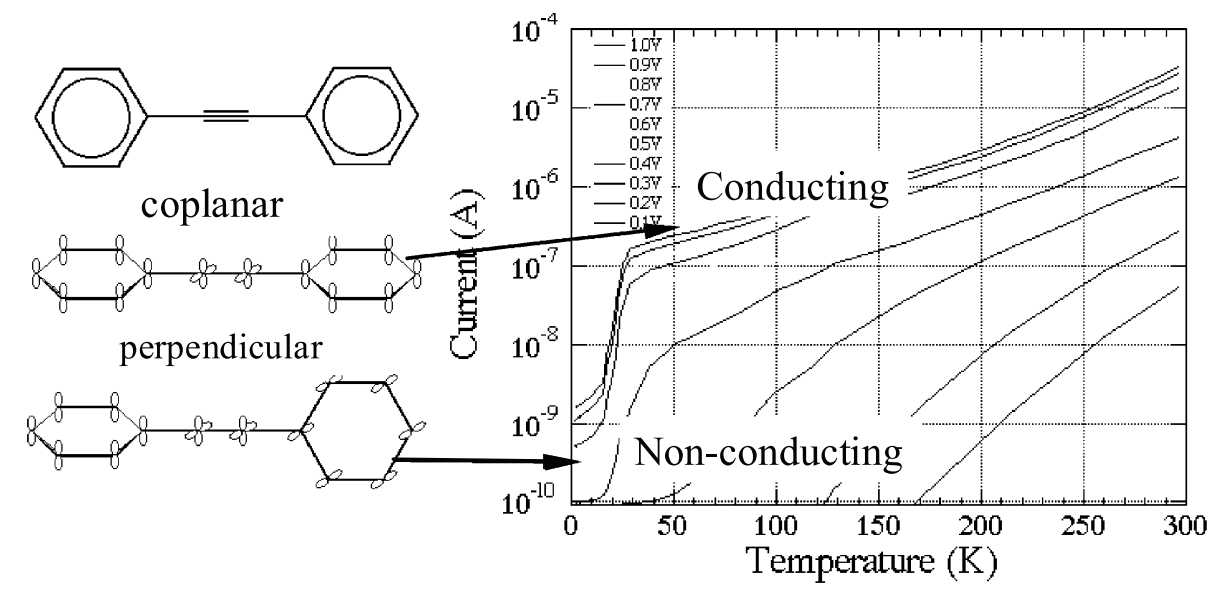

Fig. 1. I-T characteristics at different biases $(0.1-1 \mathrm{~V})$ of a $\mathrm{Au} / \mathrm{Ti} /$ ethyl-substituted $4,4^{\prime}$-di(phenylene-ethynylene)-benzothiolate/Au junction. A sharp decrease in conductivity is observed around $25 \mathrm{~K}$.

NDR devices such as Esaki tunnel diode [5], Gunn effect diode [6], resonant tunneling diode [7], etc., caused by various physical mechanisms. For example, Gunn effect diode, also known as transferred electron diode, is given rise to by a fieldinduced transfer of conduction band electrons from a low energy, high mobility valley to higher energy, low mobility satellite valleys [8].

NDR was first discovered by Esaki [5] in 1958 in a tunnel diode (a highly degenerate $\mathrm{p}-\mathrm{n}$ junction). The physical basis of the tunnel diode, which is also called the Esaki diode, is interband tunneling between the valence band and the conduction band.

Another type of well-known NDR device is the resonant tunneling diode (RTD). Tsu and Esaki proposed resonant tunneling shortly after molecular beam epitaxy (MBE) appeared in the field of compound semiconductor crystal growth in 1973 [7]. A RTD typically consists of an undoped quantum well layer (GaAs) sandwiched between undoped barrier layers (AlGaAs) and heavily doped emitter and collector contact regions [15]. Resonant tunneling through the double barrier structure occurs when the energy of the electrons flowing from the emitter coincides with the energy of the quantum well state (resonant state) in the quantum well. The effect of the external bias, $V$, is to sweep the alignment of the emitter and resonant states $\left(E_{0}\right)$. Thus a resonant tunneling current starts to flow when the Fermi level. $E_{\mathrm{F}}$ in the emitter reaches the resonant state.

There are also some organic switching devices that exhibit NDR behavior. Whereas their NDR behavior has been attributed to the formation of highly conducting filaments [18-20]. These filaments are formed by local joule heating which produces material rearrangement or even melting. This type of organic switching devices is insensitive to the polarity of the applied field, but suffers from some material rearrangement or damage. Besides, they also need high switching power consumption, and the type of metal that was used for contacts also plays an important role. For instance, Gundlach and Kadlec [18] have observed NDR in A1-(A1 oxide)-arachidic acid-Al (or $\mathrm{Au}$ ) junctions using L-B technique, but short circuits were observed when they used $\mathrm{Au}$ for both junction electrodes. The presence of $\mathrm{Al}$ oxide is vital for their experiment to block leakage current around domain boundaries or defects in the L-B film (with sample area of approximately $1 \mathrm{~mm}^{2}$ ). The NDR they observed is not repeatable.

Here we discuss the realization of large NDR behavior and room temperature operation in an electronic device that utilizes special molecules as the active component. Unlike in Esaki diode or in RTD, the monolayer thickness is determined by the SAM thickness, which inherently/ultimately solves 
the monolayer fluctuation problem at the interfaces. Being different from bulk organic switching devices, our nanoscale NDR devices are both reproducible and reversible [21].

A molecule containing a nitro-amine redox center (2'-amino-4-4'-di(ethynylphenyl)-5'-nitro-1benzenethiolate) is used in the active SAM in the nanopore configuration discussed previously. Its structure is illustrated in Fig. 2. Apart from the ethynylphenyl based backbone, there is a redox center introduced in the middle benzene ring: the electron-withdrawing nitro $\left(-\mathrm{NO}_{2}\right)$ group and the electron-rich amino $\left(-\mathrm{NH}_{2}\right)$ group.

To deposit the SAM layer onto gold electrode, we transfer the prefabricated nanopores into a $0.5 \mathrm{mM} \mathrm{2}$ '-amino-4,4'-di(ethynylphenyl)-5'-nitro1-(thioacetyl)benzene (1a) [22] in tetrahydrofuran (THF) solution. The thioacetyl groups are then selectively hydrolyzed with ammonium hydroxide (concentrated aqueous $14.8 \mathrm{M} \mathrm{NH}_{4} \mathrm{OH}, 5 \mu \mathrm{L}$ per $\mathrm{mg}$ of la) in THF to yield the free thiol, $2^{\prime}$-amino4,4'-di(ethynylphenyl)-5'-nitro-1-benzenethiol (1b), which then forms the thiolate, $2^{\prime}$-amino-4,4'-(diethynylphenyl)-5'-nitro-1-benzenethiolate (1) upon exposure to $\mathrm{Au}$ after $48 \mathrm{~h}$ [23] under an inert atmosphere of Ar. The sample was then prepared and characterized as discussed previously.

A device containing a SAM of conjugated molecules similar to $\mathbf{1}$ but not bearing the nitroamine functionalities was fabricated and measured in nearly identical conditions [4] and did not exhibit any NDR behavior. Therefore, the nitroamine redox center is responsible for the NDR behavior.

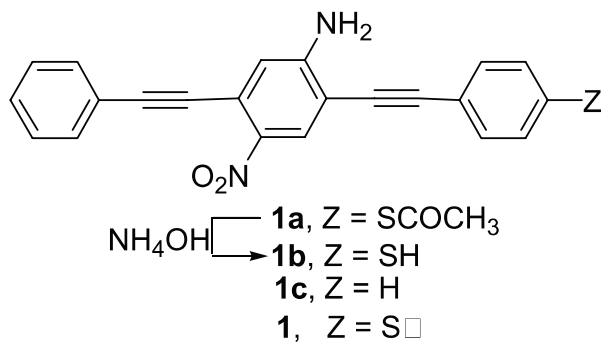

Fig. 2. The structures of active molecular compound $\mathbf{1}$, and its precursors, the free thiol $\mathbf{1 b}$ and the thiol-protected system $\mathbf{1 a}$.

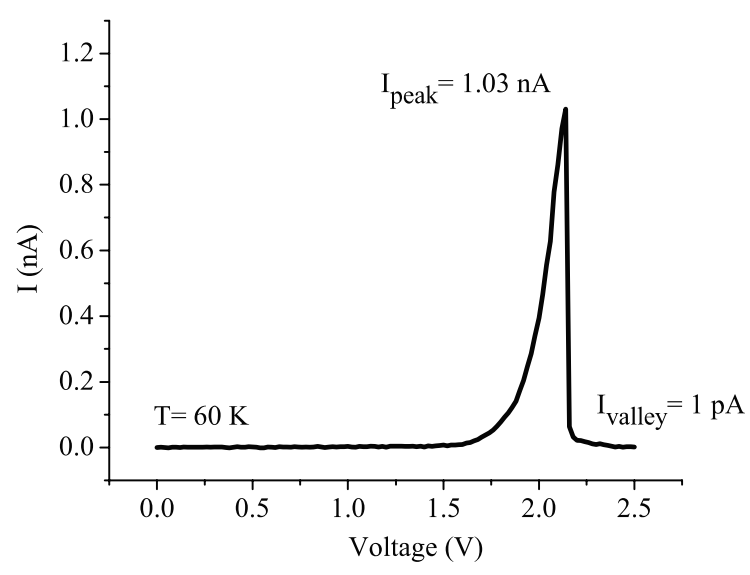

Fig. 3. $I(V)$ characteristics of a Au-1-Au device at $60 \mathrm{~K}$. The peak current density is $\sim 50 \mathrm{~A} / \mathrm{cm}^{2}$, the NDR is $\sim-400 \mu \Omega$ $\mathrm{cm}^{2}$, and the PVR is $1030: 1$.

Typical $I(V)$ characteristics of a $\mathrm{Au}-(\mathbf{1})-\mathrm{Au}$ device at $60 \mathrm{~K}$ are shown in Fig. 3. Positive bias corresponds to hole injection from the chemisorbed thiol-Au contact and electron injection from the evaporated contact. The peak current density for this device was greater than $53 \mathrm{~A} / \mathrm{cm}^{2}$, the NDR is less than $-380 \mu \Omega \mathrm{cm}^{2}$, and the PVR is 1030:1. Unlike previous devices that also used molecules to form the active region [24], this device exhibits a robust and large NDR. Some device-to-

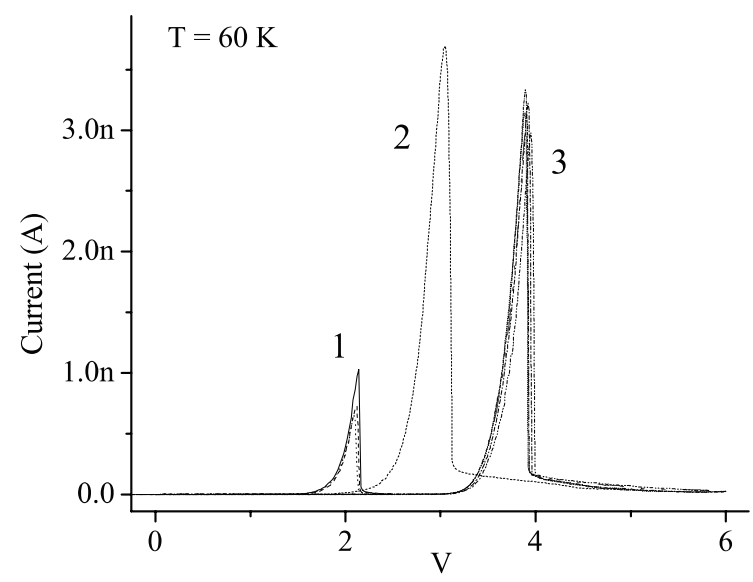

Fig. 4. $I(V)$ characteristics of $\mathrm{Au}-\mathbf{1 c}-\mathrm{Au}$ devices at $60 \mathrm{~K}$. Curve clusters 1-3 are measured from different samples; while curves within one cluster are from the same sample but from different runs. 
device variations of peak voltage position $(\sim \times 2)$ and peak current $(\sim \times 4)$ were observed as shown in Fig. 4.

Curve clusters 1-3 are from three different devices (numbered 1-3, respectively) on two different batches of wafers fabricated. Curves within each cluster are from the same device but from different runs at different time. Device-to-device current fluctuations can be attributed to fluctuations in the pore diameter size. As total voltage applied to the molecular junction can be divided into the voltage drop on the molecules and the voltage drop on the metal-molecule contacts, device-to-device peak voltage fluctuations can be caused by different voltage drops on the metal-molecule contacts. As shown by Di Ventra et al. [25], the detailed metalmolecule contact configuration plays an important part in the conductance of metal-molecular junction. Different contact geometries caused by differences in fabrication processes, such as in etching and metallization, could result in different contact potentials. Fig. 4 suggests that the voltage drop on the metal-molecule contacts on device 3 is almost twice as much as that on device 1. Further finetuning of the fabrication processes should improve uniformity and reduce device-to-device fluctuation.

The $I(V)$ curve is fully reversible upon change in bias sweep direction (from negative bias to positive bias) as shown in Fig. 5; for a given device, small fluctuations ( $\sim 1 \%$ in voltage peak position and $\sim 6 \%$ in peak current) are observed with consecutive positive and negative sweeps but could be attributed to temperature fluctuations of $\sim 2 \mathrm{~K}$ (within the experimental thermal stability). The performance exceeds that observed in typical solid state quantum well resonant tunneling heterostructures [26-29]. In addition to the obvious size advantages for scaling, the intrinsic device characteristics (that is, the valley current shutoff) may be superior to that of solid state devices. The intrinsic PVR of the molecule may be considerably greater than that reported here because the valley currents observed (on the order of picoamperes) are comparable to typical leakage currents in the silicon nitride film. At negative bias, when electrons were injected from the chemisorbed contact, the current level is approximately one order of magnitude smaller than that of positive bias as shown in Fig. 6. Negative
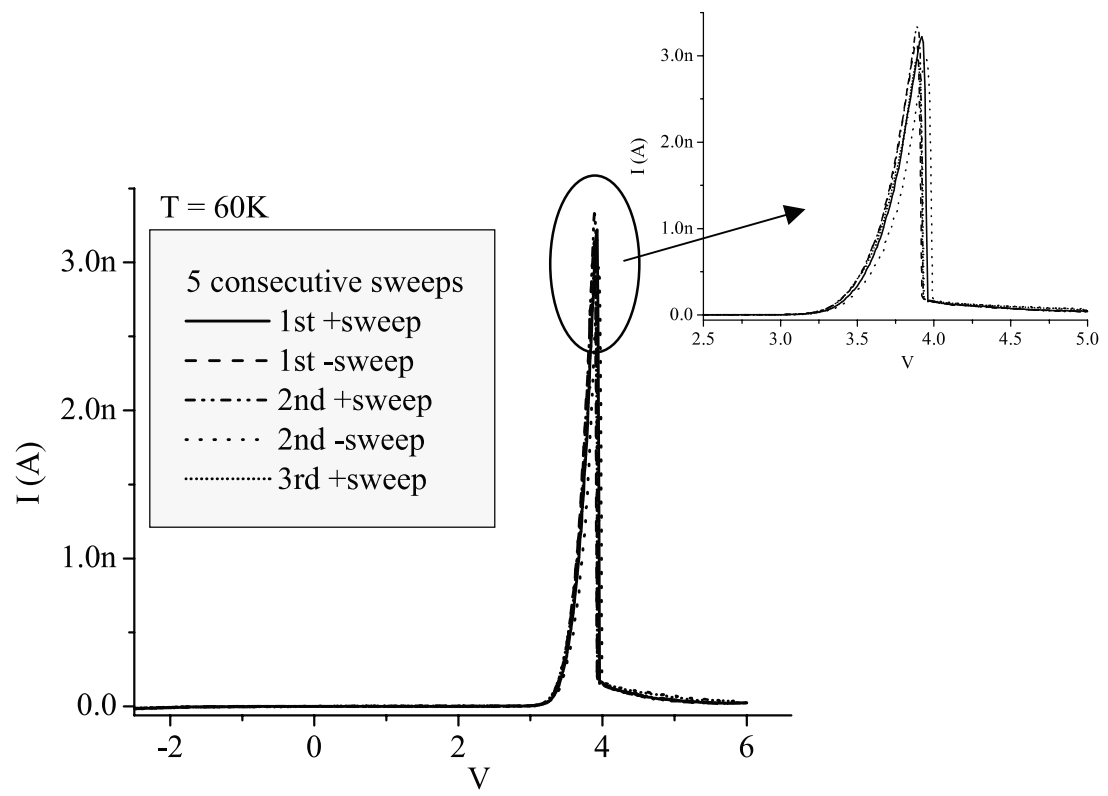

Fig. 5. Forward $(-2 \rightarrow 6) \mathrm{V}$ and reverse $(6 \rightarrow-2) \mathrm{V}$ sweeps on $\mathrm{Au}-\mathbf{1 c}-\mathrm{Au}$ junction at $60 \mathrm{~K}$. The inset is a magnification of the sweeps around the peak. 

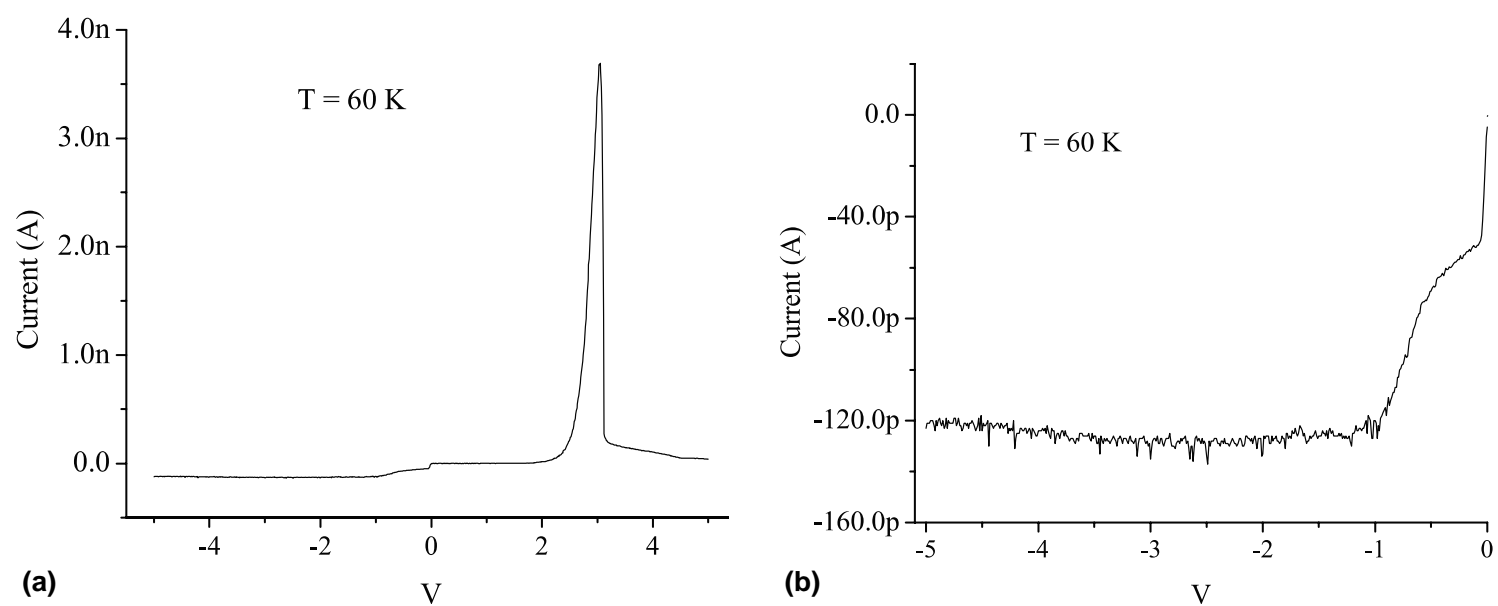

Fig. 6. Full $(-5,5) \mathrm{V}$ and negative $(0,-5) \mathrm{V}$ sweeps of a $\mathrm{Au}-\mathbf{1 c}-\mathrm{Au}$ junction at $60 \mathrm{~K}$.

bias corresponds to electron injection from the chemisorbed thiol-Au contact, whose contact barrier is around $0.7 \mathrm{eV}$, larger than the evaporated contact.

The rectifying behavior $\left(I_{+}: I_{-}=30: 1\right)$ is most likely caused by the asymmetric metal-molecular contacts.

We would like to point out the details of $I(V)$ measurements as follows: if a positive voltage sweep (referred to as $\mathrm{S1}^{+}$, e.g., from 0 to $2.5 \mathrm{~V}$ ) is followed by a negative sweep (referred to as $\mathrm{S}^{-}$, e.g., from 0 to $-2.5 \mathrm{~V}$ ), the following positive sweep (referred to as $\mathrm{S}^{+}$, from 0 to $2.5 \mathrm{~V}$ ) reproduces $\mathrm{S}^{+}$very well. If a sweep is started from negative bias to positive bias (e.g., from -2.5 to $2.5 \mathrm{~V})$, it always overlaps with consecutive sweeps, as shown in Fig. 5. If $\mathrm{S}^{+}$is followed immediately by a positive sweep (i.e., $\mathrm{S} 2^{+}$), at low temperature, NDR is observed in both sweeps with less than a few percent shift in peak position and decrease in peak amplitude; at high temperature, different phenomena (memory effects) were observed and will be discussed in detail later. $\mathrm{S}^{+} \mathrm{s}$ can be reproduced after negative sweeps $\left(\mathrm{S}^{-} \mathrm{s}\right)$. In this section, we concentrate on the investigation of NDR behavior. The positive curves shown here are $\mathrm{S}^{+} \mathrm{s}$. The behavior of $\mathrm{S}^{+} \mathrm{s}$ is very similar to that of $\mathrm{S}^{+} \mathrm{S}$ at low temperatures. In addition, $\mathrm{S}^{+} \mathrm{S}$ and $\mathrm{S}^{+} \mathrm{s}$ have very similar temperature dependences which will be shown.

\section{Temperature dependence}

All of the Au-1c-Au devices examined exhibit peak voltage position and current magnitude shifts with temperature such as shown in Fig. 7. We observe a decrease in peak intensity with increasing temperature. This could be caused by scattering in the junction. However, the fact that the peak intensity has a maximum at $60 \mathrm{~K}$ is not understood yet. It could be caused by thermally activated conformation change in the SAM layer. We

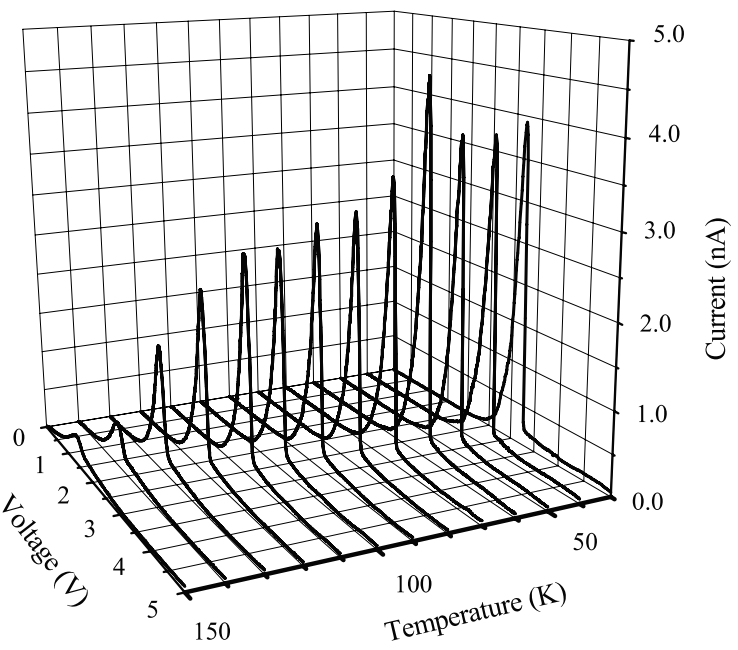

Fig. 7. $I(V, T)$ characteristics of a $\mathrm{Au}-\mathbf{1 c}-\mathrm{Au}$ device. 


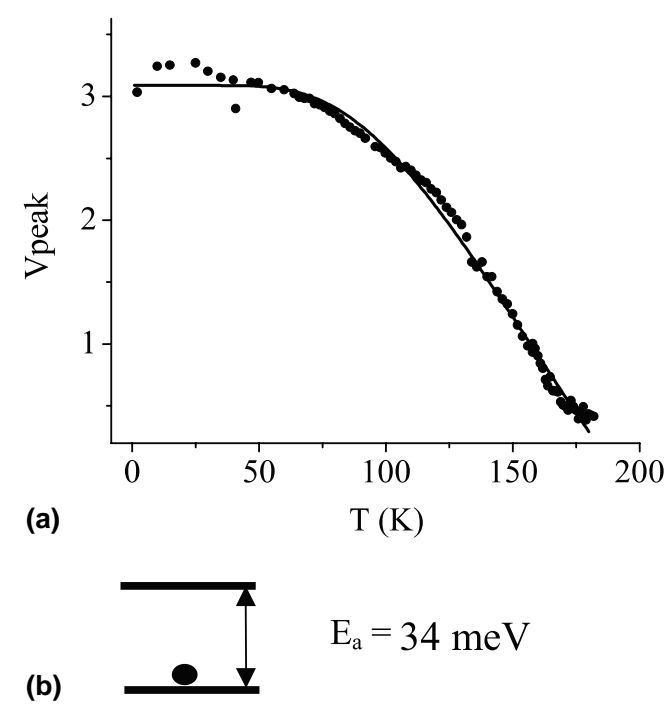

Fig. 8. (a) Peak voltage vs. temperature relationship of the same device; (b) possible physical picture of the trap state.

also observe that the peak position shifts to smaller voltage with increasing temperature (Fig. 8(a)). The shift can be fit by the following expression:

$\Delta V_{\text {peak }}=\frac{c_{1}}{1+\mathrm{e}^{-34(\mathrm{meV}) / k T}}$.

This expression can be explained by a two energy level model using a Boltzmann distribution. Where the $34( \pm 0.7) \mathrm{meV}$ corresponds to the activation barrier from the bound state as proposed in Fig. $8(\mathrm{~b})$, and $C_{1}$ is a proportionality constant. Another supporting evidence for the above model comes from the temperature dependence of peak width (Fig. 7). It remains constant at low temperatures and widens at higher temperature. Further discussions on temperature dependencies and comparison with other compounds will be carried on next.

Similar NDR behavior is also observed in devices with nitro only moiety (2). The PVR is smaller than that of $\mathbf{1}$, but NDR behavior persisted from low temperature to room temperature. $I(V)$ characteristics of a $\mathrm{Au}-(\mathbf{2})-\mathrm{Au}$ device at $300 \mathrm{~K}$ is shown in Fig. 9(a). The device has $300 \mathrm{~K}$ characteristics of a peak current density greater than $16 \mathrm{~A} / \mathrm{cm}^{2}$, NDR smaller than $-144 \mu \Omega \mathrm{cm}^{2}$ and a PVR of 1.5:1. At $190 \mathrm{~K}$ (Fig. 9(b)), the NDR peak is much sharper than that of $\mathbf{1}$, although its PVR is not as big as that of $\mathbf{1}$. The degradation in PVR (decreasing in peak current) can probably be caused by increased inelastic scattering with increasing temperature. Fig. 10 shows the cyclic voltammagram curve for compound $\mathbf{2 c}$ where the reduction potentials peaked at -1.39 and $-2.09 \mathrm{~V}$, respectively. The potentials are shifted about $0.3 \mathrm{~V}$ less than that of the nitro-amino device.

The next experiment that naturally follows is the electronic transport and electrochemistry experiments of amino only molecule $\left(2^{\prime}\right.$-amino- $4,4^{\prime}-$ di(ethynylphenyl)-1-benzenethiolate, compound
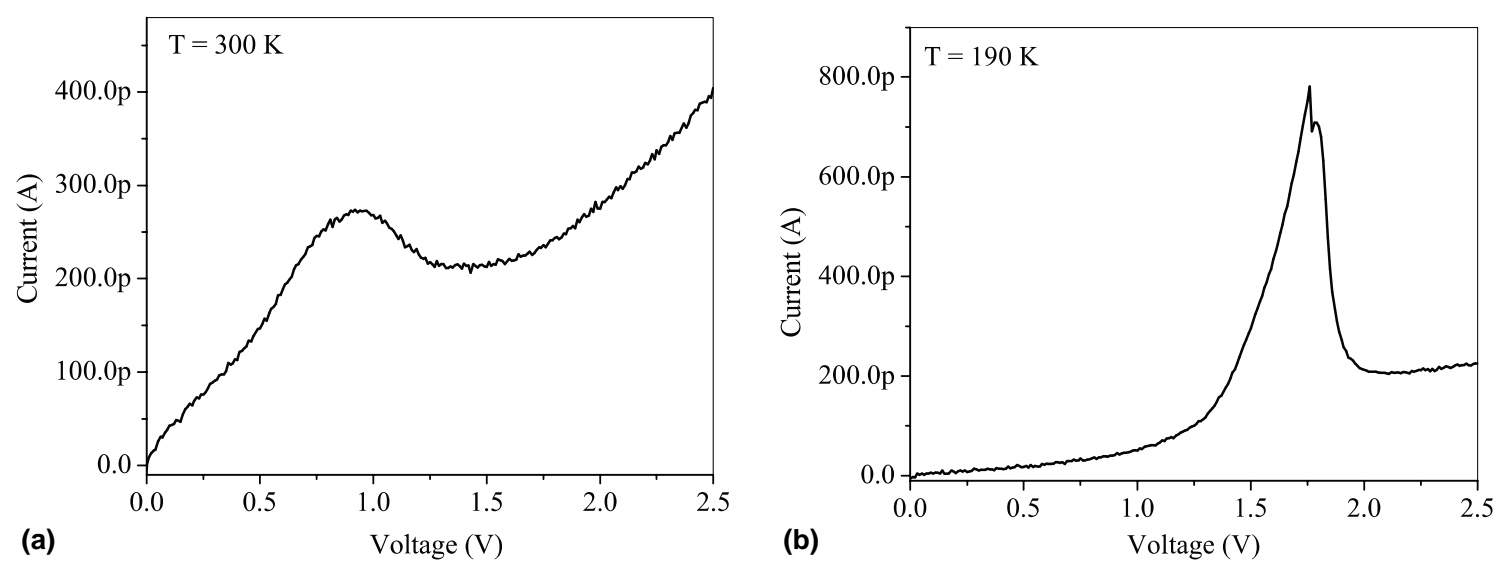

Fig. 9. $I(V)$ characteristics of a Au-2-Au device at: (a) $300 \mathrm{~K}$; (b) $190 \mathrm{~K}$. 


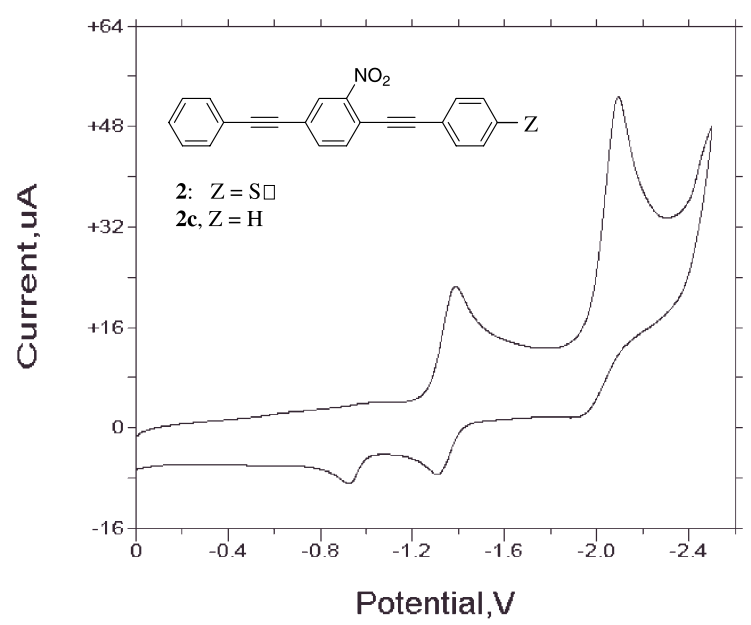

Fig. 10. Cyclic voltammagram of compound $2 \mathbf{c}$ shows two distinct reduction peaks at -1.39 and $-2.09 \mathrm{~V}$.

3). We observe no NDR behavior in 3 at both low temperature $(60 \mathrm{~K})$ and room temperature, as shown in Fig. 11. To avoid breakdown of the molecular junction, bias at room temperature was restricted to $1 \mathrm{~V}$ to limit current through the junction. There is no reduction peak in the range of interest. Concluding from above experimental results, we suggest that the nitro group is responsible for the NDR behavior. Further understanding of the underlying mechanism and experimentation

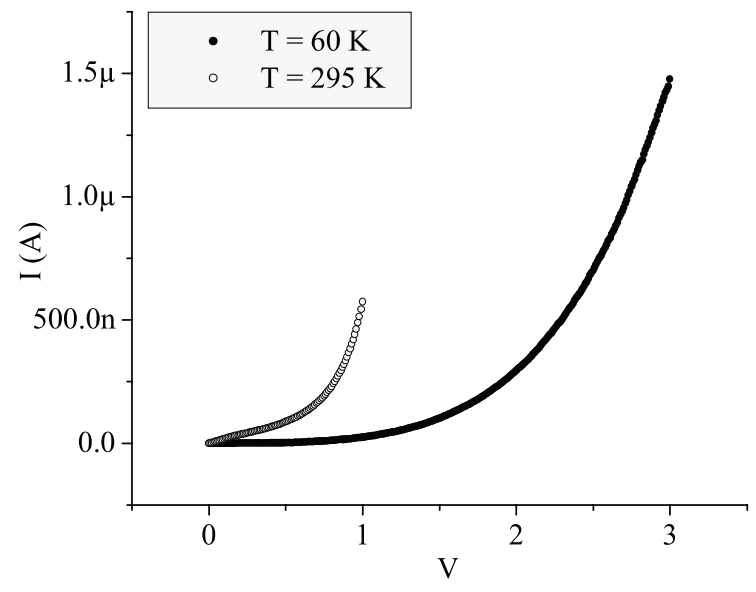

Fig. 11. $I(V)$ characteristic of $2^{\prime}$-amino-4,4'-di(ethynylphenyl)1-benzenethiolate (3) at 60 and $295 \mathrm{~K}$. No NDR behavior was observed. Bias across 3 was restricted to $1 \mathrm{~V}$ at room temperature to avoid junction break down. with various redox centers should allow us to engineer molecular compounds in the future to improve PVR at room temperature and above.

Shown in Fig. 12 is the temperature dependence of peak positions of a $\mathrm{Au}-\mathbf{2}-\mathrm{Au}$ device. The peak voltage is observed to drop linearly with increasing temperature: $\Delta V_{\text {peak }}=-0.025 \Delta T$. It appears to be very different from that of $\mathrm{Au}-\mathbf{1 c}-\mathrm{Au}$ device. To compare possible effects of different redox centers on temperature dependence, we show in Fig. 13 a plot of temperature vs. voltage values at a constant current of $10 \mathrm{nA}$ of a $\mathrm{Au}-\mathbf{3}-\mathrm{Au}$ device. Interestingly, it can also be fit to a two-level model:

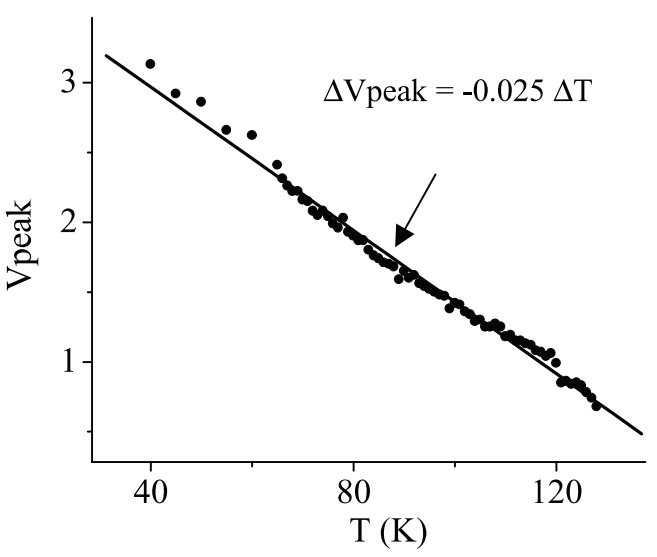

Fig. 12. Peak voltage vs. temperature of a $\mathrm{Au}-\mathbf{2}-\mathrm{Au}$ device.

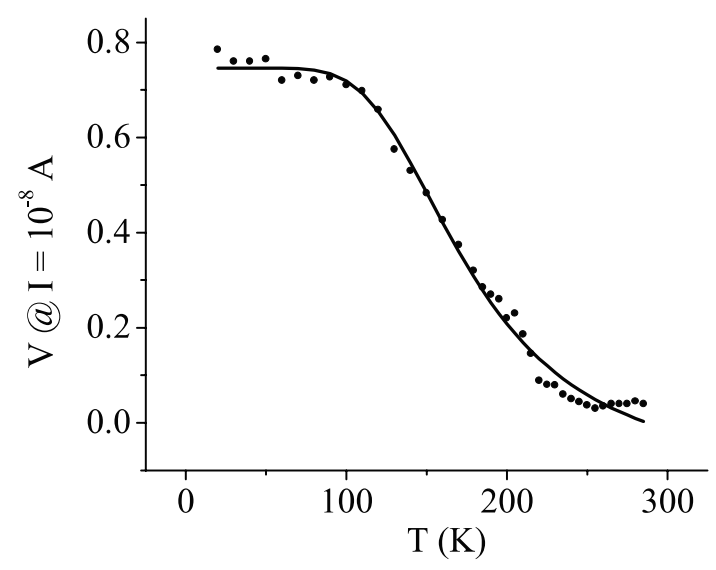

Fig. 13. Voltage values at a constant current of $10 \mathrm{nA}$ vs. temperature of a $\mathrm{Au}-\mathbf{3}-\mathrm{Au}$ device. 
$\Delta V_{@ 10 \mathrm{nA}}=\frac{c_{3}}{1+\mathrm{e}^{-30(\mathrm{meV}) / k T}}$,

where $C_{3}$ is a proportionality constant and the binding energy for the trap state on $\mathbf{3}$ is around 30 $( \pm 0.2) \mathrm{meV}$. On the other hand, the transition region of $V$ peak vs. $T$ of nitro-amine redox molecule (Fig. 7(a)) can be fit in piecewise to linear relationship as shown in Fig. 14(a), with
$\Delta V=-0.014 \Delta T$ and $\Delta V=-0.034 \Delta T$, respectively; and the transition region of $V @ 10 \mathrm{nA}$ vs. $T$ of the amine-only moiety can also be fit to linear relationship as shown in Fig. 14(b), with $\Delta V=-0.005 \Delta T$. The fitting results and comparison between compounds $\mathbf{1 c}, \mathbf{2}$ and $\mathbf{3}$ of sweeps $\mathrm{Sl}^{+} \mathrm{s}$ are summarized in Table 1. As mentioned previously and shown in Table 2, the temperature
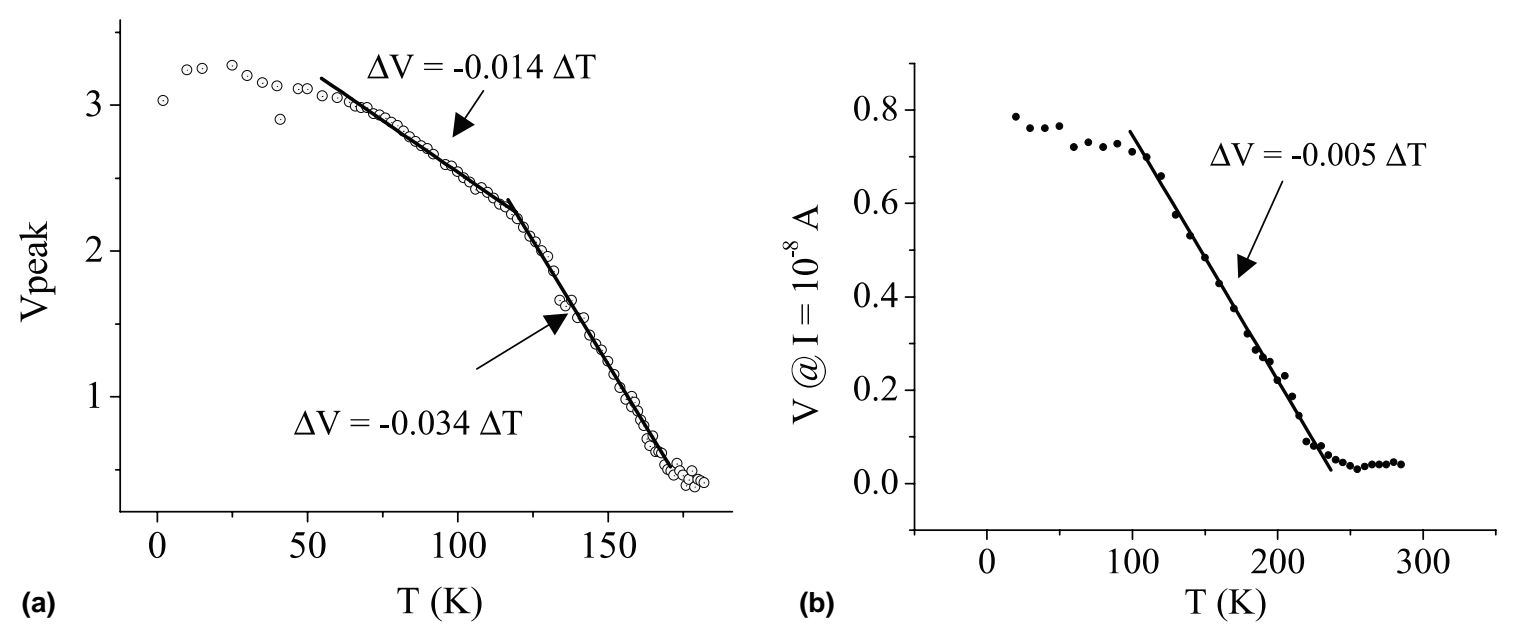

Fig. 14. Piecewise linear fitting in the transition region of: (a) peak voltage vs. temperature of a Au-1c-Au device; (b) voltage values at a constant current of $10 \mathrm{nA}$ vs. temperature of a $\mathrm{Au}-\mathbf{3}-\mathrm{Au}$ device.

Table 1

Comparison between compund 1c, $\mathbf{2}$ and $\mathbf{3}$ on temperature dependence of sweep $\mathrm{Sl}^{+} \mathrm{s}$

\begin{tabular}{lllll}
\hline Redox center & NDR & $V$ vs. $T$ & \multirow{2}{*}{$E_{\text {binding }}$} & Piecewise linear fit \\
\cline { 2 - 4 } & & Best fit & $\Delta V_{\text {peak }}=-0.025 \Delta T$ & $34 \mathrm{meV}$ \\
\hline Nitro-only (2) & $\sqrt{ }$ & $\Delta V_{\text {peak }}=\frac{c_{1}}{1+\mathrm{e}^{-34(\mathrm{meV}) / k T}}$ & $\Delta V=-0.014 \Delta T$ & $\Delta V=-0.034 \Delta T$ \\
Nitro-amine (1c) & $\sqrt{ }$ & $\Delta V_{@ 10 \mathrm{nA}}=\frac{c_{3}}{1+\mathrm{e}^{-30(\mathrm{meV}) / k T}}$ & $\Delta V=-0.0005 \Delta T$ & $30 \mathrm{meV}$ \\
\hline
\end{tabular}

Table 2

Comparison between compund 1c, $\mathbf{2}$ and $\mathbf{3}$ on temperature dependence of sweep $\mathrm{S}^{+} \mathrm{s}$

\begin{tabular}{lllll}
\hline Redox center & NDR & $V$ vs. $T$ & & $E_{\text {binding }}$ \\
\cline { 3 - 4 } & & Best fit & Piecewise linear fit & \\
\hline Nitro-only (2) & $\sqrt{ }$ & $\Delta V_{\text {peak }}=-0.005 \Delta T$ & & $32 \mathrm{meV}$ \\
Nitro-amine (1c) & $\sqrt{ }$ & $\Delta V_{\text {peak }}=-0.010 \Delta T$ & $\Delta V=-0.02 \Delta T$ & $30 \mathrm{meV}$ \\
Amine-only (3) & $\times$ & $\Delta V_{\text {peak }}=\frac{c_{1}}{1+\mathrm{e}^{-34}(\operatorname{mev}) / k T}$ & $\Delta V=-0.005 \Delta T$ & $\frac{c_{3}}{1+\mathrm{e}^{-30}(\mathrm{meV}) / k T}$ \\
\hline
\end{tabular}


dependence of sweep $\mathrm{S}^{+} \mathrm{s}$ is very similar to that of sweeps $\mathrm{S1}^{+} \mathrm{s}$. The difference has to do with the memory effects that will be discussed next.

The above result suggests that the presence of the amine group gives rise to a bound state around $30 \mathrm{meV}$ in the molecule, whereas the nitro group is responsible for the NDR behavior. The exact mechanism of linear shift of peak position with temperature is not understood at this stage yet. However, we provide one (although we stress not unique or unambiguously proven) possible explanation as follows.

The peak position shift with temperature could be caused by different vibrational modes excited at different temperatures. These vibrational modes can cause instantaneous dipole changes in the molecule which in turn interacts with electrons passing through the molecule, changing the transmission coefficient. The nitro-amine substituents in $\mathbf{1}$ have non-zero dipole moments. For instance, in nitromethane (Fig. 15), the nitrogen atom has four bonds rather than the usual three and must be represented as having a formal positive charge. The singly bonded oxygen atom, by contrast, has one bond rather than the usual two and must be represented as having a formal negative charge. The dipole moment of nitromethane resulting from the above mentioned polar bonds is $3.46 D$, where $D=3.336 \times 10^{-30} \mathrm{C} \mathrm{m}$ [30]. On the other hand, the lone pair in amine group can also lead to dipole moment. The dipole moment of ammonia $\left(\mathrm{NH}_{3}\right)$ is $1.47 \mathrm{D}$. The nitro substitutent on the benzene ring withdraws electrons from the aromatic ring by resonance (overlap of a p orbital on the substituent with a $\mathrm{p}$ orbital on the aromatic ring). Conversely, the amine substituent donates electrons (through lone pair) to the aromatic ring by resonance. With a dipole moment of $3.8 \mathrm{D}$ between nitro-amine group [31], the energy of the dipole on 1 under an electrical field of $10 \mathrm{MV} / \mathrm{cm}$ is

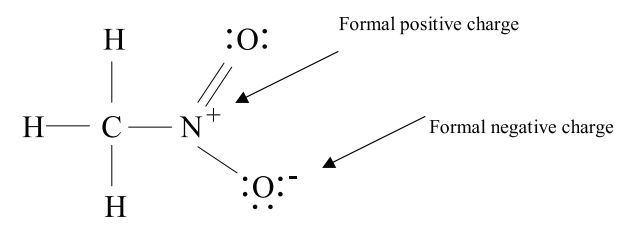

Fig. 15. Nitromethane and its dipole. approximately $40 \mathrm{meV}$, comparable to the energy scale observed in our experiment. Rotation is therefore possible. Similar rotation of benzene ring caused by nitro-amine dipole interaction has been observed by others [32]. Further IR spectroscopy experiments on 1 should help to elucidate this point.

It has been known that different rotational conformations of a molecule with the same chemical composition leads to different HOMOLUMO gap. For instance, in ethane $\left(\mathrm{CH}_{3}-\mathrm{CH}_{3}\right)$, the lowest-energy, most stable conformation is a staggered configuration where all six carbon-hydrogen bonds are as far way from each other as possible; whereas the highest-energy, least stable conformation is an eclipsed one where the six carbon-hydrogen bonds are as close as possible. Between these two limiting conformations are an infinite number of other possibilities. Experiments show that the energy barrier to rotation is $12 \mathrm{~kJ} /$ $\mathrm{mol}$ [33]. The $12 \mathrm{~kJ} / \mathrm{mol}$ of extra energy present in the eclipsed conformation of ethane is also called torsional strain which is believed to be due to the slight repulsion between electron clouds in the carbon-hydrogen bonds as they pass by each other at close quarters in the eclipsed conformer. As a result of this, the HOMO-LUMO gap energy of these two configurations are different, with a 10.99 $\mathrm{eV}$ gap of staggered ethane and $10.94 \mathrm{eV}$ of the eclipsed conformer. A much bigger effect is observed in nitramide [33], where the rotational barrier is about $48 \mathrm{~kJ} / \mathrm{mol}$ causing a change in the HOMO-LUMO gap of about $0.99 \mathrm{eV}$. Thus, a possible mechanism is that the dipole induced by the excited vibrational modes rotates the molecule under electric field, thereby changing the position of the NDR peak.

Based on our experiments, the electron-withdrawing nitro group is responsible for NDR behavior, whereas the electron-donating amine group gives rise to a bound state in the molecule of approximately $30 \mathrm{meV}$. We have learned that conformational change (rotation) and/or charging can change the conjugation of molecular orbitals. Further exploration on design and engineering of molecules with various redox substituents should help to realize non-linear electronic devices with multiple functionalities. 


\section{Molecular memory effects}

The programmable storage of digital information as packets of charge is beginning to reach not only technological but also fundamental limits. Electronic memories that operate at the charge limit (e.g., by single electron effects) have been demonstrated [34,35], but have not yet addressed the dimensional limit; i.e., a single molecule. Although memory phenomena has been studied in bulk organic materials (such as organometallic charge-transfer complex salts [1]), we will demonstrate nanoscale electronically programmable and erasable memory devices utilizing molecular SAM; and a memory cell applicable to a random access memory (RAM).

Fig. 16 lists the molecules used in this study. (They were introduced previously; we list them again for convenience.) The four systems studied are: $\mathrm{Au}-(\mathbf{1})-\mathrm{Au}$ (1: 2'-amino-4,4'-di(ethynylphenyl)-5'-nitro-1-benzenethiolate); $\mathrm{Au}-(\mathbf{2})-\mathrm{Au} \quad(2$ : 4,4'-di(ethynylphenyl)-2'-nitro-1-benzenethiolate); $\mathrm{Au}-$ (3)-Au (3: 2'-amino-4,4'-di(ethynylphenyl)-1benzenethiolate); as well as $\mathrm{Au}-(4)-\mathrm{Au}$ (4: 4,4'di(ethynylphenyl)-1-benzenethiolate) that had neither the nitro nor amine functionalities.

The memory device operates by the storage of a high or low conductivity state. Fig. 17 shows the
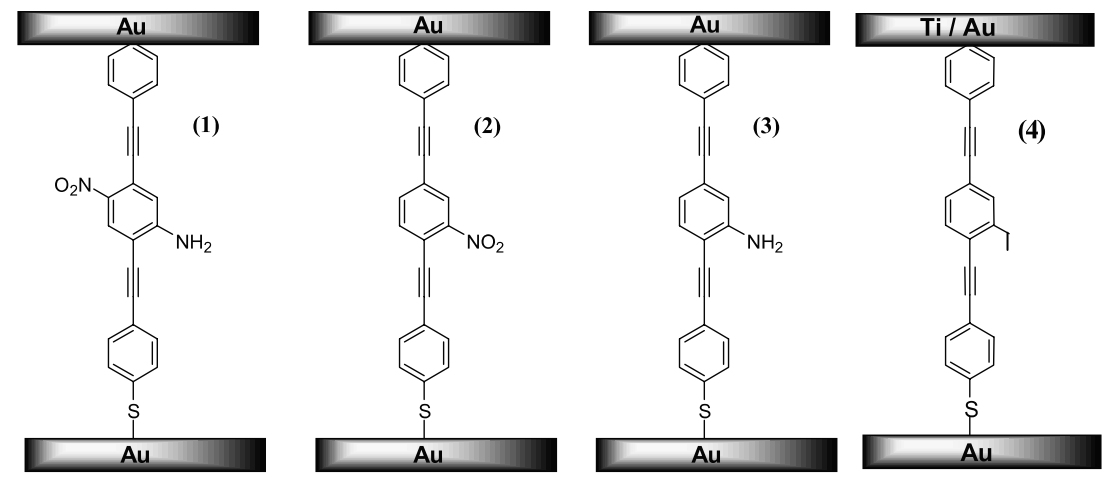

Fig. 16. The molecular junctions (1-3) used in this study. Junction 4 was studied by Zhou [4], drawn here for comparison purpose. There are approximately 1000 molecules sandwiched between the two Au contacts. Only one molecule is drawn for simplicity.

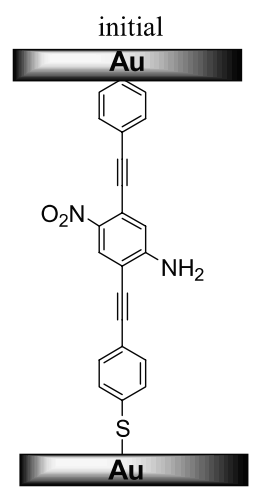

low $\sigma$

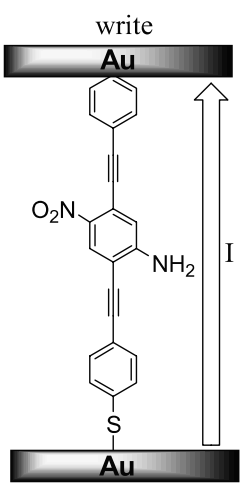

high $\sigma$

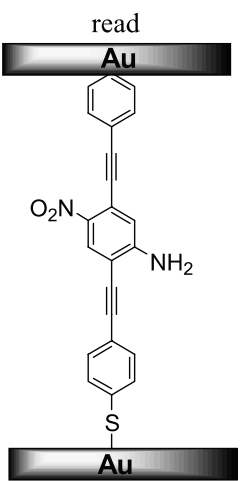

high $\sigma$

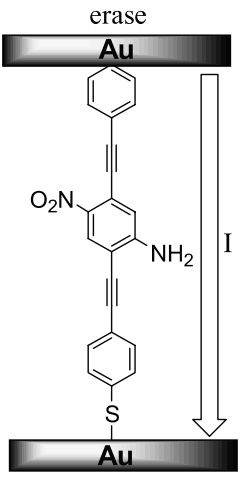

low $\sigma$

Fig. 17. The operation principle of the storage and memory. The memory device operates by the storage of a high or low conductivity state. An initially low conductivity state (low $\sigma$ ) is changed into a high conductivity state (high $(\sigma)$ upon application of a voltage. The direction of current that flows during the "write" and "erase" pulses are diagrammed by the arrows. The high $\sigma$ state persists as a stored "bit". 
write, read, and erase sequences for (1). An initially low conductivity state (low $\sigma$ ) is changed (written) into a high conductivity state (high $\sigma$ ) upon application of a voltage pulse. The direction of current that flows during this "write" pulse is diagrammed. The high $\sigma$ state persists as a stored "bit", which is unaffected by successive read pulses. Molecules with the nitro moieties (1 and $\mathbf{2}$ ) are observed to change conductivity state, whereas the amine only (3) and the unfunctionalized molecule (4) do not exhibit storage. In the following, we first describe the characteristics obtained by linear voltage sweeps (so as to generate $I(V)$ characteristics). Second we demonstrate the same effects and a circuit using voltage pulses.

Fig. 18 shows the $I(V)$ characteristics of a $\mathrm{Au}-$ (1)-Au device at $200 \mathrm{~K}$ initially (defined as " 0 ") and after (defined as " 1 ") a write pulse, as well as the difference between the two (defined as " 1 " - " $0 ")$. Positive bias corresponds to hole injection from the chemisorbed thiol-Au contact. The device initially probed with a positive voltage sweep from 0 to $2 \mathrm{~V}$ in 1 min exhibits a low conductivity state. Subsequent positive sweeps show a high conductivity state with $I(V)$ characteristics identical to the previous values (" 1 "). Device bias swept in the reverse bias direction from 0 to $-2 \mathrm{~V}$ in 1 min causes the $I(V)$ to be identically reset to the initial, " 0 " $I(V)$ characteristic. The characteristics are repeatable to high accuracy and device

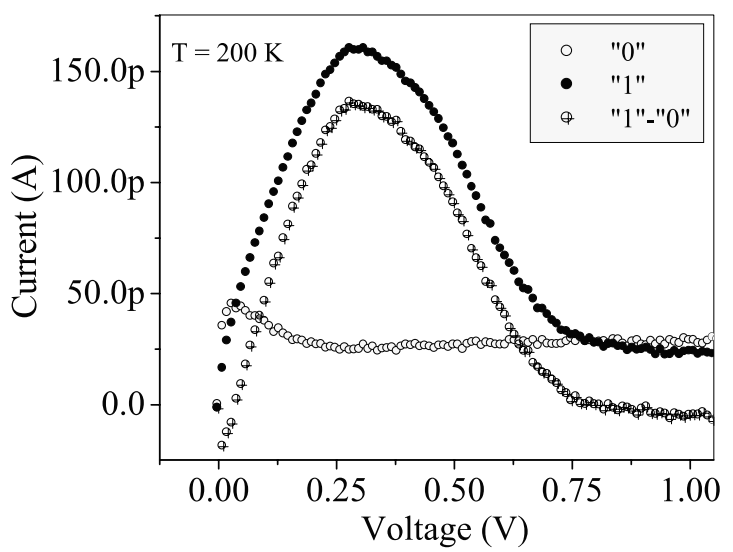

Fig. 18. $I(V)$ characteristics of a $\mathrm{Au}-(\mathbf{1})-\mathrm{Au}$ device at $200 \mathrm{~K}$. " 0 " denotes the initial state, " 1 " the stored written state, and " 1 " - " 0 " the difference of the two states.

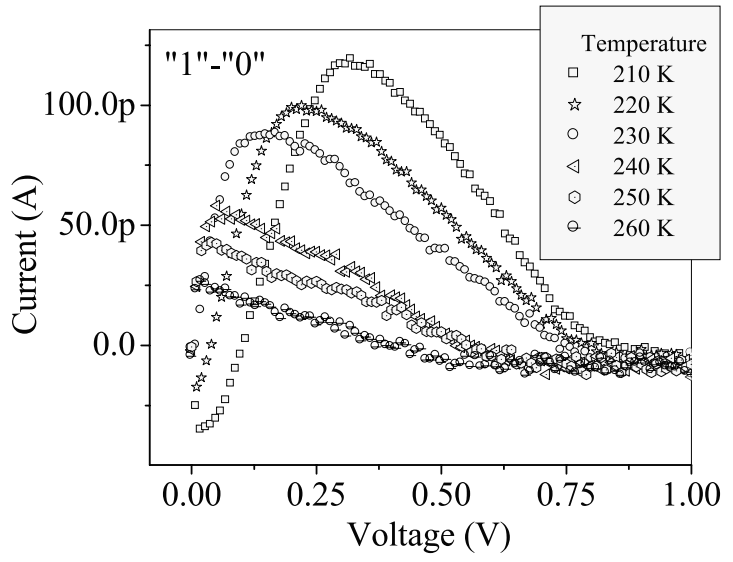

Fig. 19. Difference $I(V)$ characteristics ("1" - "0") of a $\mathrm{Au}-$ (1)-Au device as a function of temperature.

degradation is not observed. This ability to program, read, and refresh the state of the molecular device accomplishes the functionality of a RAM. Fig. 19 shows the difference characteristic ("1" - " 0 ") of (1) as a function of temperature. The peak current difference decreases approximately linear with increasing temperature.

A characteristic bit retention time was obtained by measuring the stored high conductivity state at various times intervals after programming the $\mathrm{Au}-$ (1)-Au device. After an initial positive write sweep from 0 to $2 \mathrm{~V}$ in $1 \mathrm{~min}$, a second sweep was measured at different time intervals, and the difference between the first and the second sweeps at peak current position was taken and plotted against time in Fig. 20(a). Notice that after each second sweep during the experiment, the junction has to be reset to the initial state by a negative bias sweep from 0 to $-2 \mathrm{~V}$ in $1 \mathrm{~min}$. It is found that the difference " 1 " - " 0 " exhibits an exponential decay with a time constant $(\tau)$ of approximately $800 \mathrm{~s}$ at 260 K. Similar measurements were performed from 260 to $190 \mathrm{~K}^{2}$ The stored state was found to decay exponentially with increasing time constants at lower temperatures. Shown in Fig. 20(b) is a plot of the decay time constant (retention time) at

\footnotetext{
${ }^{2}$ Below $190 \mathrm{~K}$, the $I(V)$ characteristic changes considerably, with the NDR peak shifting much toward higher voltage. No appreciable decay of current was observed.
} 

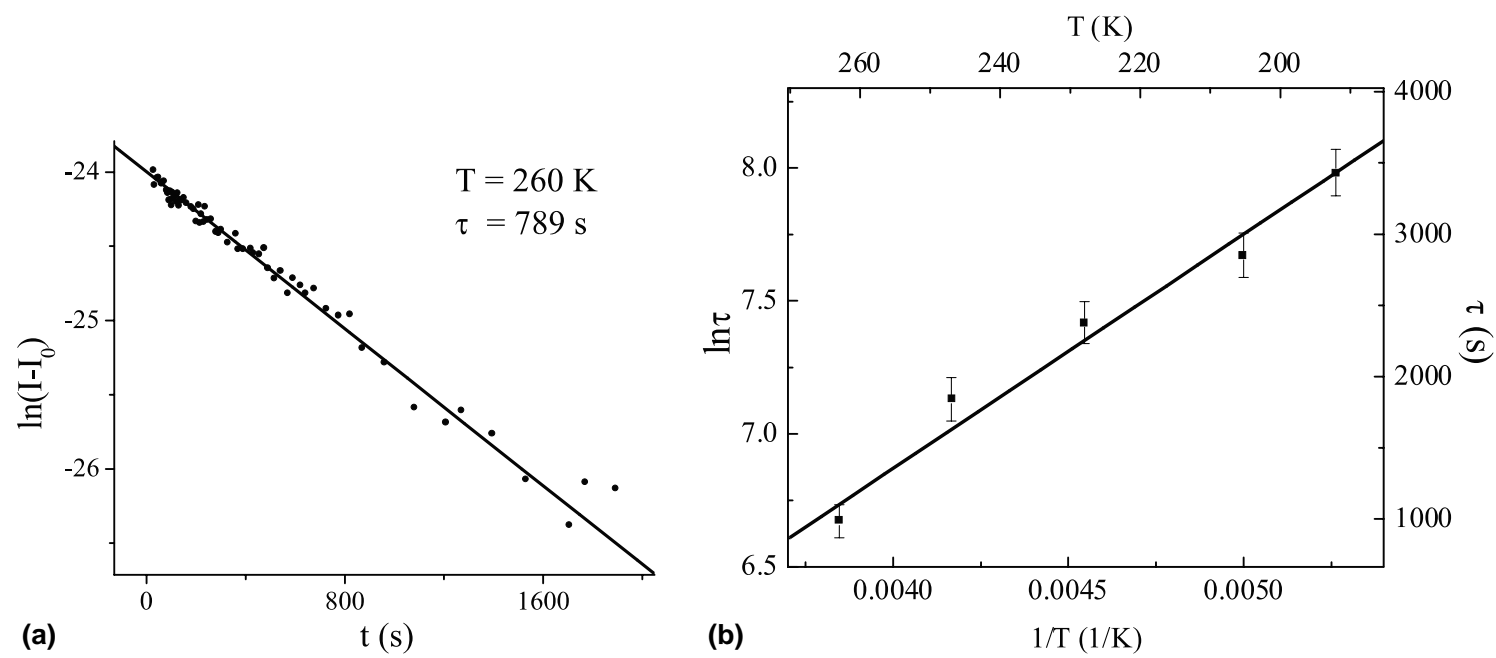

Fig. 20. Bit retention as a function of time and temperature. (a) Bit retention for (1) exhibits an exponential decay with a time constant $(\tau)$ of $790 \mathrm{~s}$ at $260 \mathrm{~K}$. (b) Temperature dependence of $\tau$ gives an activation energy $E_{\mathrm{a}}=76 \pm 7 \mathrm{meV}$.

different temperatures, exhibiting an exponential dependence with $1 / T$. It indicates an activation behavior: $\tau=\tau_{0} \exp \left(E_{\mathrm{a}} / k T\right)$, suggesting activation over a single trap state. The activation energy $E_{\mathrm{a}}$ for this molecule over this bias regime was found to be approximately $80 \mathrm{meV}$.

So far we only discussed the memory effects when the molecular junction is under positive bias (other than the negative sweep used to refresh the written state). Fig. 21(a) shows the first (" 0 ") and second (" 1 ") traces when the same junction is biased negatively $(0,-5 \mathrm{~V})$ at $100 \mathrm{~K}$. The window over which the " 0 " and " 1 " differ by a constant amount of $-120 \mathrm{pA}$ is nearly $4 \mathrm{~V}$, providing wellseparated thresholds. The third trace and so on overlap with the second one. Contrary to the positive bias, initially, the molecular junction is in a higher conductivity state, whereas it is changed to a lower conductivity. After applying a positive sweep, we observe that the junction is set back to the initial negative trace (overlaps with " 0 "). Shown in Fig. 21(b) is the difference between the first and second $I(V)$ traces (" 0 " - " 1 ") at various temperatures. Notice that the width of the plateau
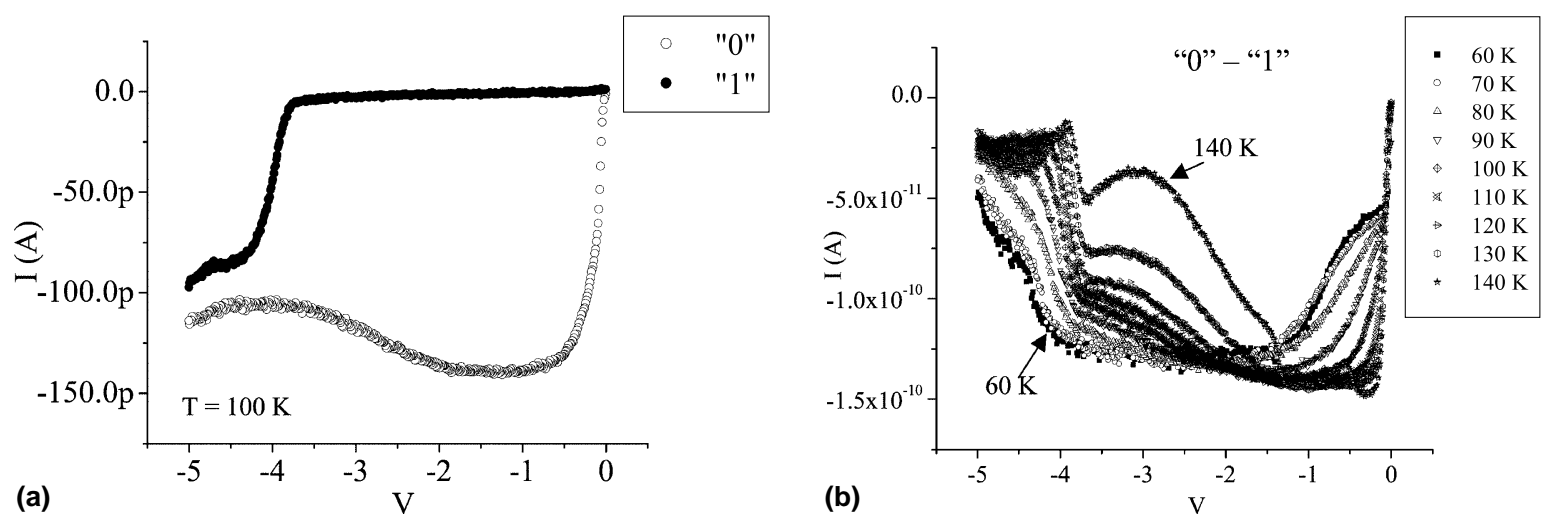

Fig. 21. (a) $I(V)$ characteristics of the first trace (erased/initial state) and the second trace (stored state) in $\mathrm{Au}-(\mathbf{1})-\mathrm{Au}$ at $100 \mathrm{~K}$ under negative bias; (b) difference $I(V)$ characteristics (" 0 " - " 1 ") at different temperatures. 

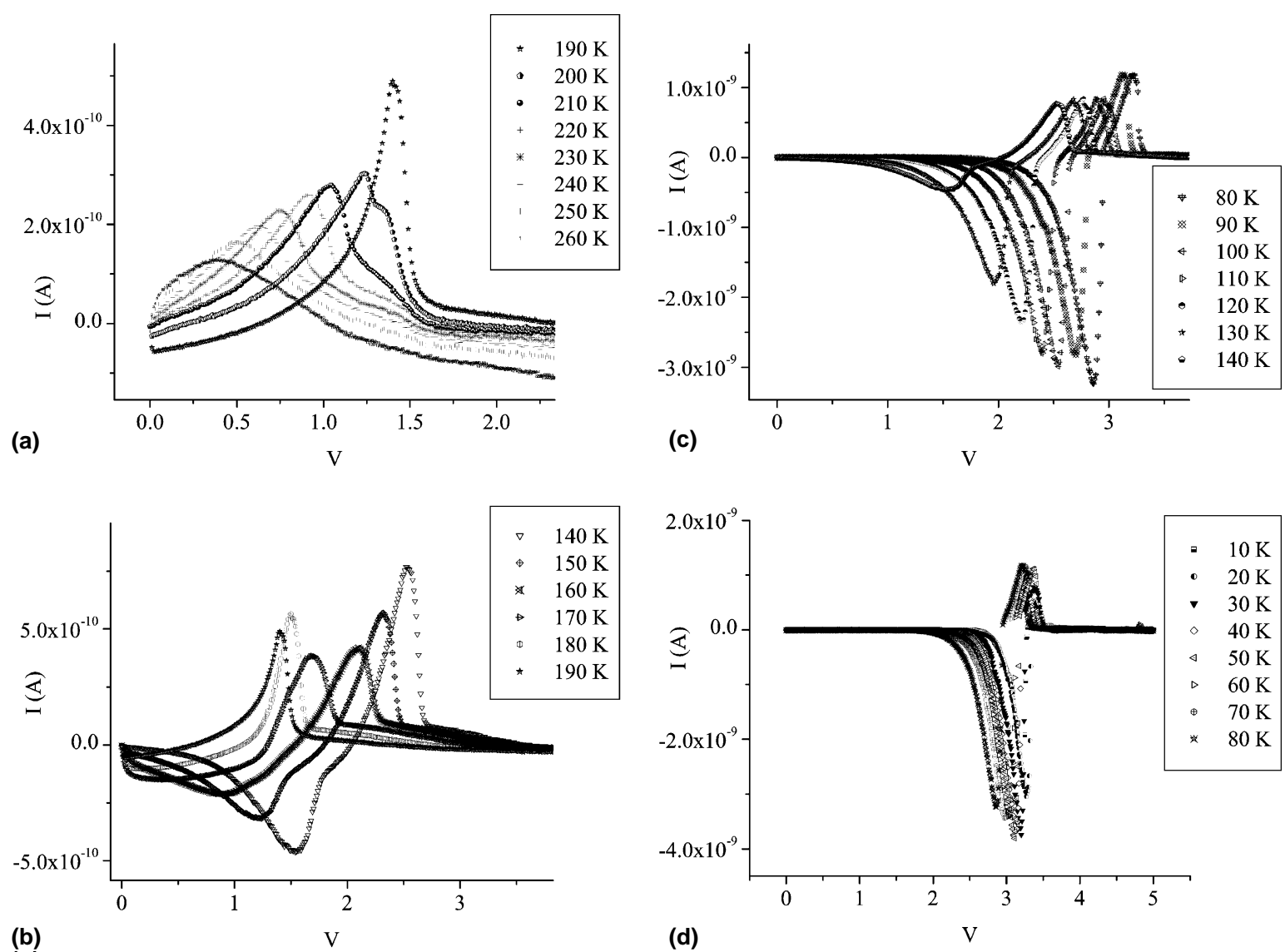

Fig. 22. Difference $I(V)$ curves ("1" - "0") between 10 and $260 \mathrm{~K}$ of a Au-(1)-Au device: (a) 190-260 K; (b) 140-190 K; (c) 80-140 K; (d) $10-80 \mathrm{~K}$.

decreases with increasing temperature. The decay of the stored state under negative bias is very slow. The decay constant is estimated to be larger than hours.

Figs. 22(a)-(d) shows the difference $I(V)$ traces ("1" - " 0 ") $)^{3}$ between 10 and $260 \mathrm{~K}$ of a Au-(1)Au device. As seen in Fig. 22(a), in addition to the major NDR peak, there is an evolution of a second peak at higher bias, which is most obvious at 200 K and disappears around 190 K. Simultaneously, the difference curve changes its sign at low bias around $200 \mathrm{~K}$, suggesting a change in the conducting states in the molecule. No appreciable

\footnotetext{
${ }^{3}$ The two traces are measured immediately one after the other.
}

decay of current was observed below $190 \mathrm{~K}$. This could possibly be caused by suppression of specific conformational modes in the molecule at low temperatures. Below $160 \mathrm{~K}$, it is seen that there are two distinct NDR peaks, one from the first trace (peaked at smaller voltage), the other from the second trace (peaked at larger voltage). Peak position difference between the two NDR peaks remains approximately the same below $80 \mathrm{~K}$. The peak amplitude decreases to roughly $1 / 3$ at the second sweep. The third traces and so on repeat the second ones. A negative sweep brings the molecular junction to its original state, after which the first traces can be reproduced. This observation could possibly be caused by excess charge trapped in the junction. 

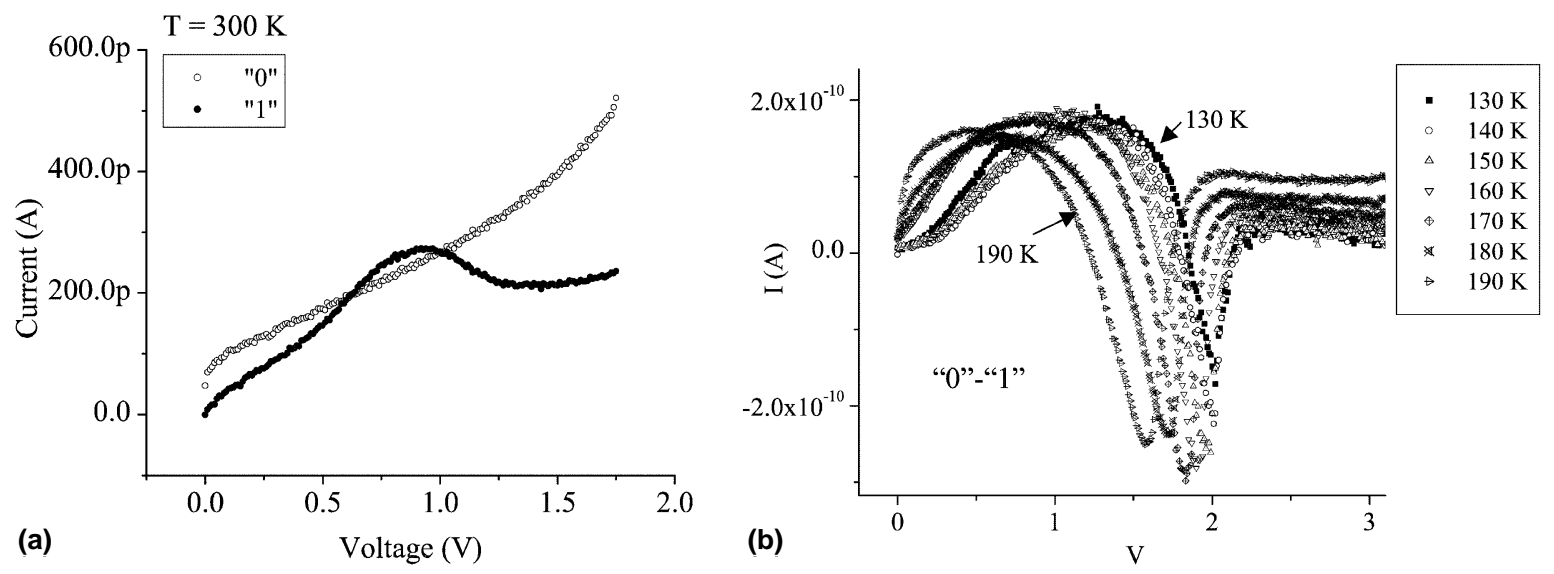

Fig. 23. (a) $I(V)$ characteristics of the first trace (initial state) and the second trace (stored state) in Au-(2)-Au at $300 \mathrm{~K}$ under positive bias; (b) difference $I(V)$ characteristics ("0" - "1") at various temperatures.

Memory effects are also observed in devices with the molecules having only the nitro moiety (2), although in this case the storage was of a low conductivity state ${ }^{4}$ after an initial positive sweep from 0 to $1.75 \mathrm{~V}$ in $1 \mathrm{~min}$, opposite to that of molecule (1). Fig. 23(a) demonstrates the storage of this state in molecule (2) at $300 \mathrm{~K}$. NDR was observed in the stored state " 1 ". As described previously, subsequent reads and resets identically recovered the $I(V)$ characteristics. At $300 \mathrm{~K}$, thermal activation does contribute some non-zero " 0 " current, although the thresholds are still well-separated (approximately $250 \mathrm{pA}$ ). Fig. 23(b) shows the difference $I(V)$ characteristics (" 0 " - " 1 ") at various temperatures, " 0 " $>$ " 1 " (before NDR region), opposite to that of molecule (1).

$I(V)$ characteristics under negative biases are shown in Fig. 24. Fig. 24(a) shows the first/initial, and second sweeps from 0 to $-5 \mathrm{~V}$ in $2 \mathrm{~min}$, respectively. As described before, subsequent reads and resets identically recovered the $I(V)$ characteristics. Fig. 24(b) shows the difference $I(V)$ sweeps ("0"- " 1 ") at various temperatures. The width of the current plateau decreases with increasing temperature, similar to that of molecule (1).

\footnotetext{
${ }^{4}$ Not including the NDR region, where current level is higher than the initial state.
}

A characteristic bit retention time is obtained by measuring the stored low conductivity state at various times intervals after programming the $\mathrm{Au}-$ (2)-Au device. After an initial write bias sweep, current $\left(I_{1}\right)$ at $1.5 \mathrm{~V}$ of the stored state " 1 " increases gradually until it recovers the initial " 0 " high conductivity state $\left(I_{0}\right)$. Their difference $\left(I_{0}-I_{1}\right)$ exhibits an exponential decay with a time constant $(\tau)$ of approximately $900 \mathrm{~s}$ at $300 \mathrm{~K}$ (Fig. 25).

Memory effects are not observed in molecule (3) with the amine-only group. Shown in Fig. 26 are typical $I(V)$ characteristics of a $\mathrm{Au}-(3)-\mathrm{Au}$ device at 60 and $295 \mathrm{~K}$, respectively. The first and second traces at a constant temperature overlap with each other. The current level is higher than that of both $\mathrm{Au}-(\mathbf{1})-\mathrm{Au}$ and $\mathrm{Au}-(\mathbf{2})-\mathrm{Au}$ devices, suggesting that the presence of nitro group impedes charge transport, causing a high conductivity state in (1) (with nitro-amine group) and a low conductivity state in (2) (with nitro-only group). Consequently, the $80 \mathrm{meV}$ energy level observed in (1) is most likely associated with the nitro group.

The above observation leads us to the following preliminary model for the memory effect (Fig. 27). We have identified from above experimental results that the nitro group is responsible for the memory effect, while the presence of the amine group changes the initial conductivity states of the 

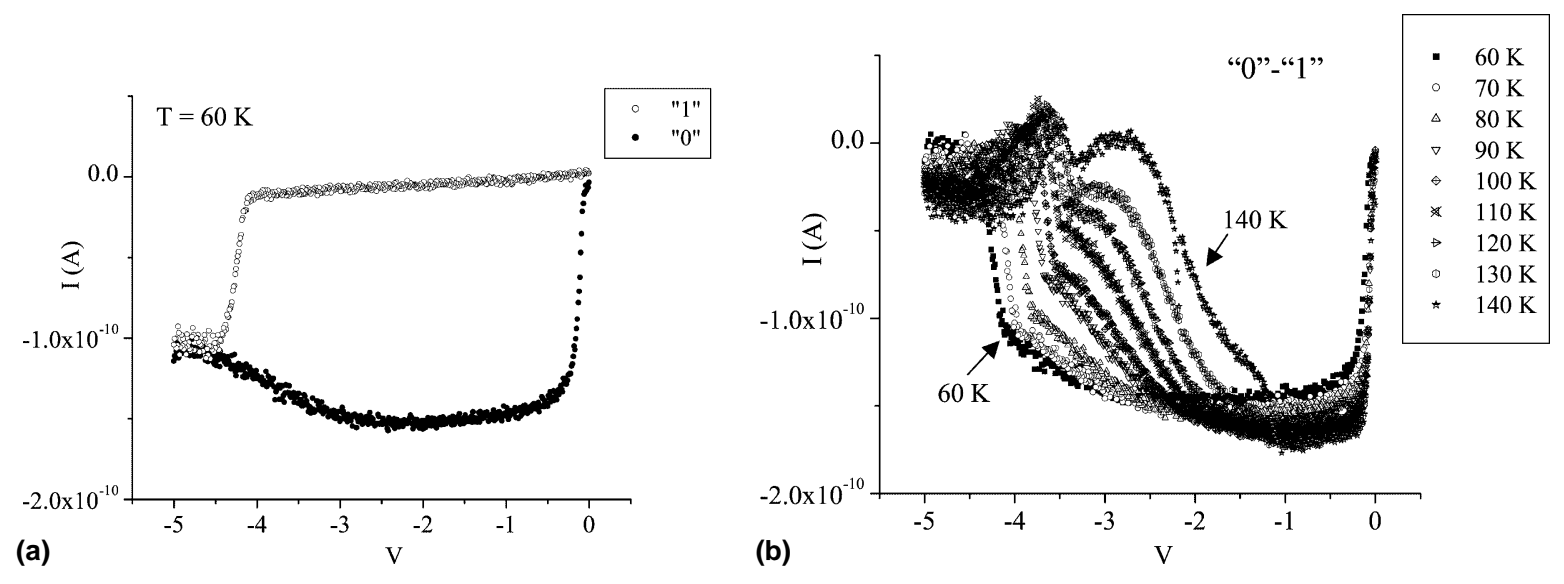

Fig. 24. (a) $I(V)$ characteristics of the first trace (initial state) and the second trace (stored state) in Au-(2)-Au at $60 \mathrm{~K}$ under negative bias; (b) difference $I(V)$ characteristics (" 0 " - "1") at various temperatures.

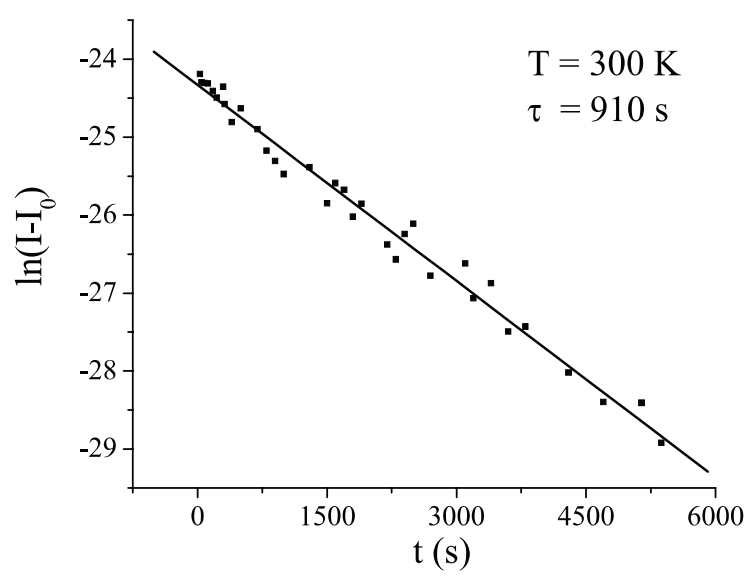

Fig. 25. Measurement of retention time for (2). Current exhibits an exponential decay with a time constant $(\tau)$ of $910 \mathrm{~s}$ at $300 \mathrm{~K}$.

molecule (most likely caused by the different dipole distribution it introduces to the molecule). Here we use molecule $\mathbf{1}$ as an example. Depending on its history, $\mathbf{1}$ can be in one of the two states, L (low conductivity state) or $\mathrm{H}$ (high conductivity state). We propose that the different conductivity states are caused by different trap or conformation levels occupied by electrons in the molecule as shown in Fig. 27(a). Different trap levels occupied by electrons in a molecule could mean either different electronic states with different

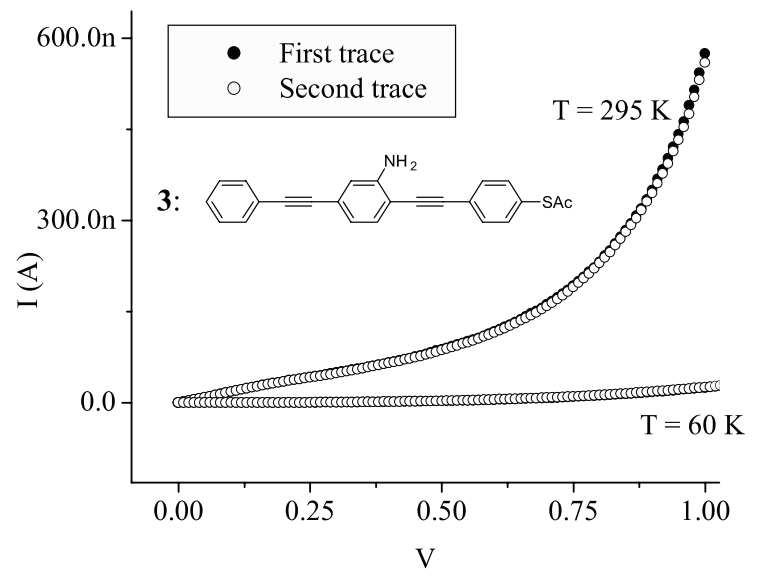

Fig. 26. $I(V)$ characteristic of $2^{\prime}$-amino-4,4'-di(ethynylphenyl)1-benzenethiolate (3) at 60 and $295 \mathrm{~K}$. First and second traces overlap with each other.

charge distribution (such as different dipole distribution) or different conformation states in a molecule (such as rotational conformations, about various axes), or both. The microscopic origin of the trap states is at this point unknown; we stress that regardless of the microscopic origin, we have determined here that the origin is a trap state, and the energetic characteristics of that trap state.

These trap levels can cause different electronic distribution, leading to various conductivity states 


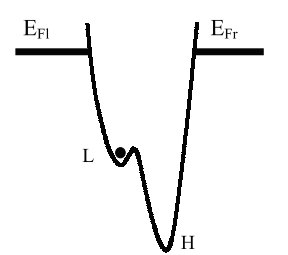

(a)

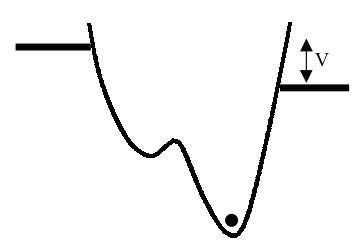

(c)

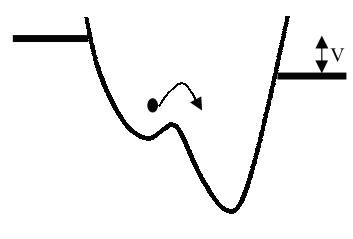

(b)

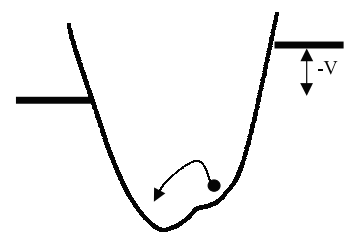

(d)
Fig. 27. Proposed model for the memory effect. Lowering of $E_{\mathrm{Fr}}$ corresponds to positive bias: (a) initial low conductivity state, zero bias; (b) first positive sweep, "write", changed into a high conductivity state $-\mathrm{H}$; (c) consecutive positive sweeps, "read", remained in the high conductivity state; (d) first negative sweep, "erase", back to low conductivity state.

of the molecule. $\mathbf{1}$ is initially in a low conductivity state (L). As the junction is biased positively, the barrier is modified, and the electron falls into state $\mathrm{H}$ (Fig. 27(b)). When the bias is removed the electron remains undisturbed in the same localized state $\mathrm{H}$ they occupied under bias for a period of time equal to $(v P)^{-1}$ where $v$ is the attempting frequency to escape and $P$ is the probability of escaping the localized state $\mathrm{H}$. In general $P$ is proportional to the Boltzmann factor $\exp (-E / k T)$, where $E$ denotes the energy barrier of the localized state $\mathrm{H}$. It corresponds to the measured $80 \mathrm{meV}$ as shown in Fig. 20. For subsequent positive biases at times $t<(v P)^{-1}$, the electron is trapped in state $\mathrm{H}$ (Fig. 27(c)). Only with negative bias can the electron be "liberated" and go back to state L (Fig. 27(d)). At times $t>(v P)^{-1}$, the electron leaks through the barrier and finally returns to state $\mathrm{L}$. The electron will be trapped in state $\mathrm{L}$ with following negative sweeps, and it can only be refreshed to $\mathrm{H}$ state by positive bias. This explains well that the first negative sweep on the junction shows a high conductivity state (Fig. 21), i.e., the initial $\mathrm{H}$ state after positive sweeps; the negative traces show low conductivity states; and a positive trace resets the state.

\section{Demonstration of a molecular memory storage cell}

To illustrate application of this memory effect, a memory circuit was constructed utilizing a $\mathrm{Au}-\mathbf{2}-$ Au device in combination with a sense circuit. The relevant part of the $I(V)$ characteristic used is shown in Fig. 28, with the setpoints marked. Because the on/off window is sufficiently large, this effectively demonstrates a molecular RAM cell at ambient temperature.

Fig. 29 shows the TTL-compatible I/Os of this circuit. The upper trace in Fig. 29 is an input waveform applied to the device, and the lower is the RAM cell output. The first positive pulse

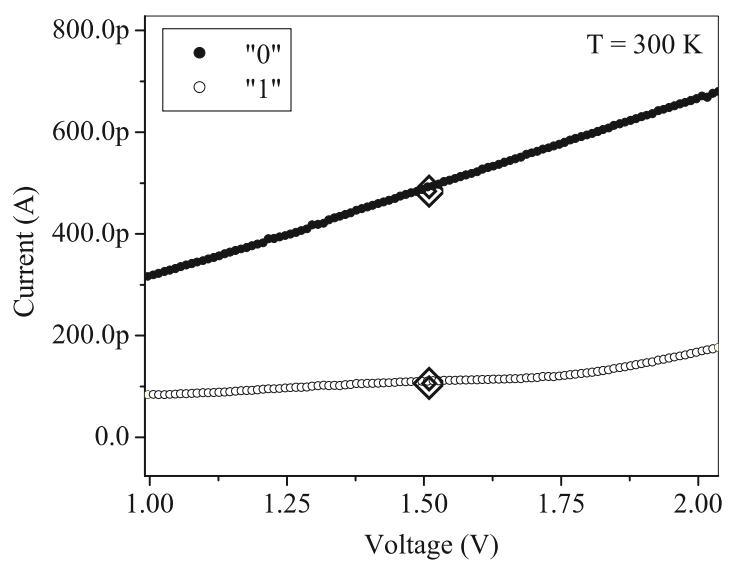

Fig. 28. $I(V)$ characteristics of stored and initial/erased states in $\mathrm{Au}-(\mathbf{2})-\mathrm{Au} 300 \mathrm{~K}$. The set-points indicated are the operating points for the circuit.

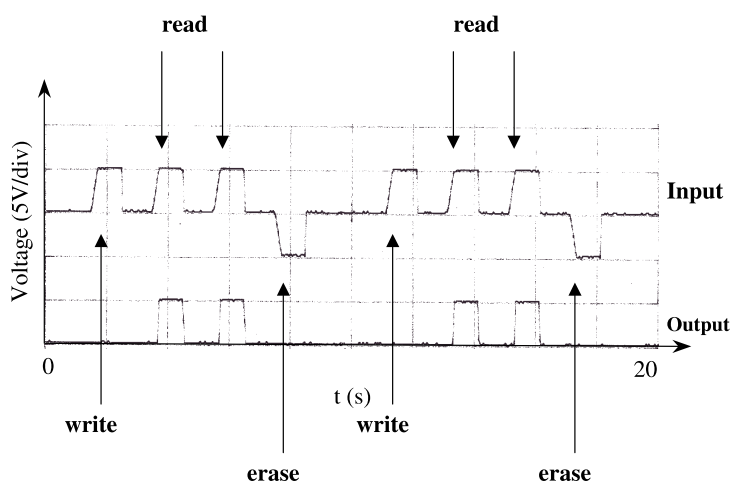

Fig. 29. Measured logic timing diagram of the molecular random access memory. 
configures the state of the cell by writing a bit, and the second and third positive pulses read the cell. The third pulse (and subsequent read pulses, not shown here for simplicity) demonstrates that the cell is robust and continues to hold the state (up to the limit of the bit retention time. This demonstration highlights the dramatically long bit retention time.). The negative pulse erases the bit, resetting the cell. The second set of four pulses repeats this pattern, and many hours of continuous operation have been observed with no degradation in performance. Currently the speed of the memory device is limited by the RC time constant of the circuit. There is a $\sim 1 \mathrm{pF}$ capacitance between the two $\mathrm{Au}$ electrodes in the nanopore configuration. The current level of the devices is less than $10 \mathrm{nA}$, which can be attributed to the 0.7 eV S-Au barrier [36,25] in the molecular junction. Engineering of molecules with smaller contact barriers (e.g., use $\mathrm{CN}$ terminated molecules and $\mathrm{Pd}$ contacts) to obtain higher current level while minimizing contact area to decrease parasitic capacitance should improve the RC limit.

The present devices utilize nanoscale structures that limit the number of molecules in the active region to $\sim 1000$, which is determined by lithographic limitations in defining the contacts. We have seen no evidences in the device characteristics indicating that limitations exist for scaling the number of molecules in the active region to one, assuming that an appropriate fabrication scheme can be identified.

\section{Summary}

Electronic transport is measured and characterized in a class of oligo-phenylene-ethynylene devices, and the relevant transport barriers and traps are elucidated. The non-linear and hysteretic behaviors observed in these devices are shown to have integrated circuit utility, and are the first steps toward future molecular electronics.

\section{References}

[1] R.S. Potember, T.O. Pochler, D.O. Cowan, Appl. Phys. Lett. 34 (1979) 405.
[2] R.S. Potember, T.O. Poehler, D.O. Cowan, A.N. Bloch, in: L. Alcbcer (Ed.), The Physics and Chemistry of LowDimensional Solids, D. Reidel Publishing Company, Dordrecht, 1980, p. 419.

[3] T. Hertel, R.E. Walkup, Ph. Avouris, Phys. Rev. B 58 (1993) 13870.

[4] C. Zhou, Thesis, Yale University, 1999.

[5] L. Esaki, Phys. Rev. 109 (1958) 603.

[6] L.B. Gunn, Solid State Commun. 1 (1963) 88.

[7] L.L. Chang, L. Esaki, R. Tsu, Appl. Phys. Lett. 24 (1974) 593.

[8] H. Kroemer, Proc. IEEE 52 (1964) 1736.

[9] T.C.L.G. Sollner et al., Appl. Phys. Lett. 43 (1983) 588.

[10] M. Tsuchiya, H. Sakaki, J. Yoshino, Jpn. J. Appl. Phys. 24 (1985) L466.

[11] S.M. Sze (Ed.), High-Speed Semiconductor Devices, Wiley, New York, 1990

[12] F. Capasso, R.A. Kiehl, J. Appl. Phys. 58 (1985) 1366.

[13] N. Yokoyama, K. Imamura, S. Muto, S. Hiyamizu, N. Nishi, Jpn. J. Appl. Phys. 24 (Part2) (1985) L583.

[14] F. Capasso, in: G.A. Mourou, D.M. Bloom, C.H. Lee (Eds.), Picosecond Electronics and Optoelectronics, Springer, Berlin, 1985, p. 112.

[15] L. Esaki, IEEE Trans. Electron Dev. 12 (1965) 374.

[16] A. Seabaugh, R. Lake, Encyl. Appl. Phys. 22 (1998) 335.

[17] H. Mizuta, T. Tanoue, The Physics and Applications of Resonant Tunneling Diodes, Cambridge, 1995.

[18] K.H. Gundlach, J. Kadlec, Phys. Stat. Sol. A 10 (1972) 371.

[19] C. Hamann et al., Phys. Stat. Sol. A 50 (1978) K189.

[20] A.R. Elsharkawi, K.C. Kao, J. Phys. Chem. Solids 38 (1977) 95.

[21] J. Chen, M.A. Reed, A.M. Rawlett, J.M. Tour, Science 286 (1999) 1550.

[22] The starting compound (1a) was prepared by sequential $\mathrm{Pd} / \mathrm{Cu}$-catalyzed coupling of 2,5-dibromo-4-nitroacetanilide with phenylacetylene and 4-ethynyl(thioacetyl)benzene.

[23] J.M. Tour et al., J. Am. Chem. Soc. 117 (1995) 9529.

[24] Weak room temperature NDR has been previously reported M.A. Reed, Proc. IEEE 87 (1999) 652.

[25] M. Di Ventra, S.T. Pantelides, N.D. Lang, Phys. Rev. Lett. 84 (2000) 979.

[26] J.H. Smet, T.P.E. Broekaert, C.G. Fonstad, J. Appl. Phys. 71 (1992) 2475.

[27] J.R. Söderström, D.H. Chow, T.C. McGill, J. Appl. Phys. 66 (1989) 5106.

[28] J. Day et al., J. Appl. Phys. 73 (1993) 1542.

[29] H.H. Tsai et al., IEEE Elec. Dev. Lett. 15 (1993) 357.

[30] J. McMurry, in: Organic Chemistry, Brooks/Cole Publishing Company, 1996, p. 108.

[31] Private communication with A. G. Zacarias.

[32] H.J. Gao et al., Phys. Rev. Lett. 84 (2000) 1780. 
[33] J.M. Seminario, P. Politzer, Int. J. Quantum Chem. S26 (1992) 497.

[34] S. Tiwari, F. Rana, H. Hanafi, A. Hartstein, E.F. Crabbe, K. Chan, Appl. Phys. Lett. 68 (1996) 1377.
[35] A single electron memory operating at $4 \mathrm{~K}$ was demonstrated in N.J. Stome H. Ahmed, Elec. Dev. Lett. 20 (1999) 583.

[36] M.A. Reed et al., Science 278 (1997) 252. 